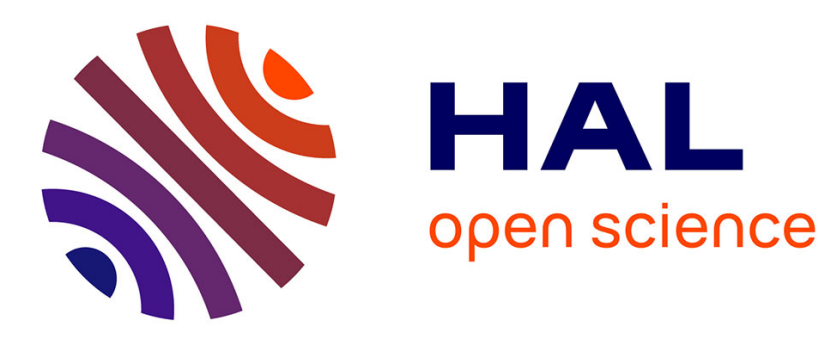

\title{
High System Capacity Pre-Scheduler for Multi-Cell Wireless Networks
}

Nicolas Guérin, Malo Manini, Rodolphe Legouable, Cédric Gueguen

\section{To cite this version:}

Nicolas Guérin, Malo Manini, Rodolphe Legouable, Cédric Gueguen. High System Capacity Pre-

Scheduler for Multi-Cell Wireless Networks. Wireless Networks, 2021, 27, pp.13-25. hal-03125029

\section{HAL Id: hal-03125029 \\ https://hal.science/hal-03125029}

Submitted on 29 Jan 2021

HAL is a multi-disciplinary open access archive for the deposit and dissemination of scientific research documents, whether they are published or not. The documents may come from teaching and research institutions in France or abroad, or from public or private research centers.
L'archive ouverte pluridisciplinaire HAL, est destinée au dépôt et à la diffusion de documents scientifiques de niveau recherche, publiés ou non, émanant des établissements d'enseignement et de recherche français ou étrangers, des laboratoires publics ou privés. 


\title{
High System Capacity Pre-Scheduler for Multi-Cell Wireless Networks
}

\author{
Nicolas Guérin, Malo Manini, Rodolphe Legouable, Cédric Gueguen \\ $\mathrm{b}<>$ com, University of Rennes 1/IRISA \\ Emails: \{nicolas.guerin, malo.manini, rodolphe.legouable\}@b-com.com, cedric.gueguen@irisa.fr
}

\begin{abstract}
With the constant increase of throughput demands, maximizing the wireless network capacity has always been a crucial issue. Network densification becomes one of the adopted strategies to guarantee a high Quality of Experience (QoE). These new access points are included in the traditional 5G coverage scheme and offload the network in dense areas where the demand is high. A mobile may be located in the coverage area of several base stations which implies the need to extend traditional scheduling to a multicell context. This leads to the emergence of new issues concerning resource allocation strategies. When one user is suitable to receive resources from different cells, a well designed users' distribution is essential to limit unbalanced and overloaded cells. The main contribution of this paper is to propose a pre-scheduler called Multi-Cell Pre-Scheduler (MCPS) that adequately allocates user traffic between different access points, intervening before the scheduling application. Compatible with any type of simple cell existing schedulers, MCPS adapts schedulers to multi-cell context and improves their performance by delaying the system congestion and making the system more robust to unexpected traffic peaks. Performance evaluations show that the use of MCPS solution allows to increase the throughput capacity and energy efficiency while improving QoE fairness between users.
\end{abstract}

Index Terms-Wireless Network, Resource allocation, PreScheduling, Multi-Cell, 5G.

\section{INTRODUCTION}

With the emergence of new services (video streaming, web, IoT...) requiring more and more throughput and low latencies, guaranteeing a good QoE becomes really challenging. Indeed, network resources such as bandwidth are often scarce and need to be shared between users inside the same coverage area. In order to manage this traffic, network densification constitutes a major evolution in new network deployment. This lies in the multiplication of the number of access points with which users can establish a connection. In a $5 \mathrm{G}$ multicell context, we generally consider that micro cells $(\mu \mathrm{gNB})$ are deployed in addition to the traditional 5G-based macro cell (gNB) [1]. These cells allow to locally unload the system in dense areas where the demand in data may be higher (shopping centers, stadiums, train stations). Access points are then divided into two categories whose characteristics are presented as follows:

- A macro cell access point provides a wide-area coverage due to their low frequencies band $(<6 \mathrm{GHz})$, facilitating a geographical continuous connection to users. Traditionally a macro cell base station can cover up to $10 \mathrm{~km}$ in rural areas and up to $1 \mathrm{~km}$ in dense urban area.

- A micro cell access point is a low-power hotspot providing short range coverage area. Operating in high frequency bands (millimeter waves for $5 \mathrm{G}$ ), these access points allow to deliver high data throughput thanks to large spectrum bandwidth. However these frequencies suffer from strong pathloss, shadowing and multipath fading significantly affecting the coverage area.

In this context, the management of all these access points could be done by the macro cell access point. The Central Unit management (Figure 1) performs all the scheduling decisions and allocates resources to the users. This hierarchical management [2] (also called "backhaul") optimizes the decisionmaking on how the network operates and allocates resources depending on user demands and channel quality.

In this new type of wireless network, a new problematic lies in the allocation process. Indeed, in a typical 5G multicell scenario (MIMO for instance), radio layer evolution allows a user to be located in the coverage area of several access points at the same time. This undeniably leads to the emergence of new allocation strategy issues. One of them lies in optimizing user traffic repartition between micro cell and macro cell access point. This depends on a lot of parameters such as users channel quality, services type and system load. Thereafter, the study proposes different allocation strategies when the system suffers from high traffic load and tends to be overload.

A well studied topic, coordinated multipoint (CoMP) transmissions, tackles the users repartition in multi-cells context, as seen in [3] and [4]. However, our solution takes benefit from different frequency bands for each station type. The macro cell and the micro cell work at different frequency, eliminating the interference problem. Moreover, our pre-scheduler proposal is compatible with all allocation strategies (schedulers). Four of them are briefly summarize below.

This paper aims to present MCPS pre-scheduling solution and evaluate its performance impact when applied upstream these four schedulers. MCPS computes user experienced throughput with all the access points they can establish a connection. It assigns to each user a priority index. Depending on the micro cell maximal capacity, users with the higher priority index are assigned to the micro while the rest of the users are assign to the macro. This allows to assigned to the macro cell the users who are the most beneficial to it. By dynamically arrange users between the two access points, the user traffic repartition is optimized guaranteeing a high system capacity.

The paper is organized as follows. Section II describes related work concerning scheduling schemes and multi-cells algorithms. Section III provides a detailed description of the system under study and gives an important preliminary result attesting the use of a pre-scheduler. Section IV introduces 


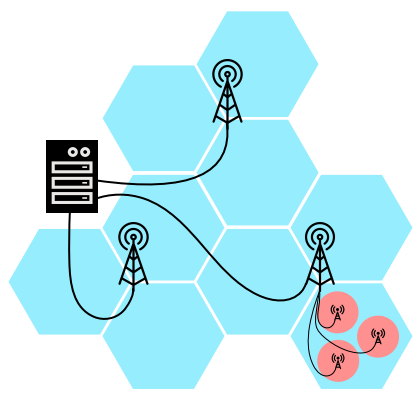

(a) Decentralized management

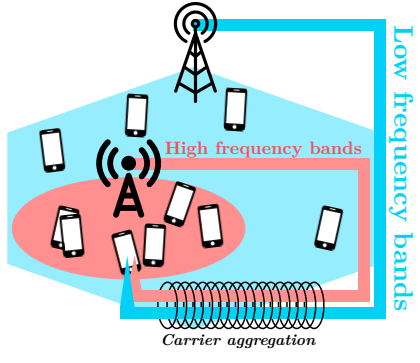

(b) User deployment
Figure 1: Typical 5G multicell scenario

MCPS pre-scheduler principles. Section V presents two users deployment scenarii in order to evaluate the performance of our pre-scheduling solution. Section VI concludes the paper.

\section{RELATED WORK}

\section{A. Scheduling schemes}

All the following scheduling schemes differ in their Key Performance Indicators (KPI) requirements and so in the way they operate. For example, a scheduler may focus on throughput maximization, fairness between users or energy saving.

- Round Robin (RR) [5] scheduler is probably one of the simplest algorithms to study and to implement. Its performance are widely known, which make this nonopportunistic scheduler a reference. Round Robin scheduler alternately assigns a resource unit to each user (Figure 2). At each decision-making instant, a given Resource Unit (RU) is firstly allocated to $\mathrm{UE}_{1}$, then to $\mathrm{UE}_{2}$, and that until $\mathrm{UE}_{\mathrm{N}}$, before come back to $\mathrm{UE}_{1}$. Originally used in wired networks, RR isn't well adapted to wireless network. Indeed it doesn't take into account users Channel State Information (CSI) which does not allow it to claim a good throughput. An allocation strategy example is given on Figure 2 (blue curve).

- MaxSNR [6] is considered as a reference in wireless resource allocation. Taking advantage of variable channel conditions and multi-user diversity, this opportunistic scheduler aims to maximize the global throughput in the system. By allocating a given resource unit (RU) to the user having the best achievable throughput on it (i.e. the best channel condition), MaxSNR scheduler firstly allocates resources to the users who is less affected by pathloss, shadowing and multipath fading. However, close users have more chance to have access to the medium. It results in a fairness deficiency between close and far users whose achievable throughput (affected by pathloss) does not allow them to have the same priority in the MaxSNR allocation process. An allocation strategy example is given on Figure 2 (red curve).

- Proportional Fair (PF) [7,8] scheduling provides similar benefit than MaxSNR concerning system capacity maximization but it ensures more fairness between all users. User priority depends on instantaneous SNR but

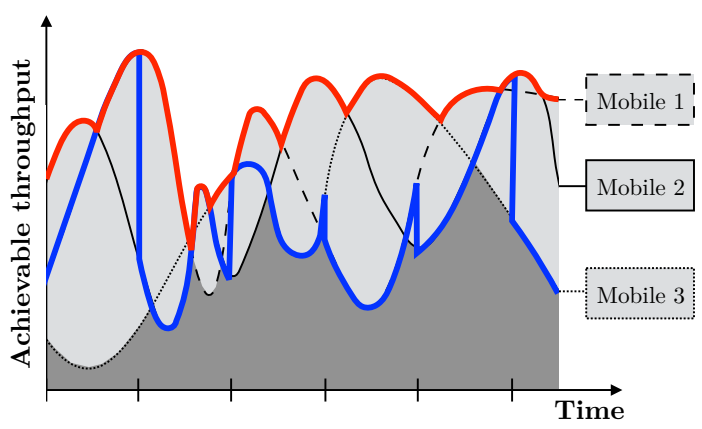

Figure 2: RR (—) and MaxSNR (-) allocation strategies

is also inversely proportional to the user mean obtained throughput. Far users with lower mean throughput obtain a higher priority which allows them to get more chance to have access to the medium. So PF equally considers all users independently of their channel conditions and benefits from a better multi-user diversity. So it admits a slight system capacity gain compared to MaxSNR.

- Opportunistic Energy Aware (OEA) scheduler [9,10] objective is to minimize energy consumption exploiting active-sleep mode and channel condition together. Like previous opportunistic schemes, this will allow to reach good system capacity. OEA determines the best transmission opportunities and finds the most profitable resource mapping in terms of number of transmitted bits per Watt. This scheduler keeps a bad fairness between users.

\section{B. Multi-cells algorithms}

Using the same frequency for all access points leads to the need of advanced interference management. Coordinated multipoint (CoMP) $[3,4,11,12]$ is a topic well covered in the literature and aims at covering this problem. The objective is to provide a fair throughput regarding the position of the users, where edge users may suffer from interference of nearby cells. In our context, edge users are covered by a micro-cell and does not suffer from interference thanks to the use of different frequencies. On the other hand, Ultra Dense small cell Network (UDN) reduces the opportunistic feature of schedulers due to very short Inter-Site Distance (ISD) as indicated in [13]. One advantage of our solution is to be compatible with different types of schedulers without changing their resource allocation policy but by managing previously to the transmission the schedule between micro and macro cells.

\section{SYSTEM DESCRIPTION}

\section{A. Deployment scenario}

In the studied deployment scenarii, the entire bandwidth is split into several frequency slots also called "subcarriers". We assume that each subcarrier has independent channel state value [14]. Depending on the numerology used, time slot duration and subcarrier sizes of this granulated time/frequency grid may differ. The packets issued from the backhaul network are buffered in the access point which schedules the downlink transmission. The radio resources are further divided in the 
time domain in frames. Each frame is itself divided in Time Slots (TS) of constant duration. The Time slot duration is an integer multiple of the OFDM symbol duration. The number of subcarriers is chosen so that the width of each sub-frequency band is less than the coherence bandwidth of the channel. Moreover, the frame duration is fixed to a value much smaller than the coherence time of the channel. We consider two access points with available Resource Units (RUs). Each Resource Unit is characterized by a \{frequency; time $\}$ pair and represents an unbreakable element which is entirely made available to a user (Figure 3). All results are obtained using a discrete events simulator written in $\mathrm{C}++$.

We assume a simple deployment scenario by considering a unique micro cell access point whose coverage area (represented in red on figures) is fully included in the macro cell one (represented in blue). We also assume that a user achievable throughput is linked to the power he receives and is limited due to his distance from the access point and multipath fading [15]. The potential number of bits that can be transmitted on a given $\mathrm{RU}$ is given by the following equation 1 corresponding to the upper bound:

$$
q_{k, n} \leq\left\lfloor\log _{2}\left(1+\frac{3 P \times T_{s} \times\left(\frac{1}{d_{k}}\right)^{3.5} \times \alpha_{k, n}^{2}}{2 N_{0}\left[\operatorname{erfc}^{-1}\left(\frac{B E R_{\text {target }, k}}{2}\right)\right]^{2}}\right)\right\rfloor
$$

where $\mathrm{P}$ is the transmit power, $N_{0}$ is the noise spectral density, $T_{s}$ the OFDM symbol duration, $d_{k}$ the distance between the user $\mathrm{k}$ and the access point, $\alpha_{k, n}$ represents the flat fading experienced by user $\mathrm{k}$ on subcarrier $\mathrm{n}$. In the following, $\alpha_{k, n}$ is Rayleigh distributed with an expectation equal to unity. The potential number of bit that a user can transmit on a RU will fluctuate around this value over the time. In the rest of the paper we consider that all users get the same $B E R_{\text {target }}$ of $10^{-3}$. We assume each base station has a full knowledge of the channel for each user.

We also consider an admission control that denies the connection between a user and an access point if its received power and therefore its achievable throughput is below a given limit. This situation may happen when a user is far from a base station and need too much RUs to guarantee a sufficient quality of experience. Each of the two access points made available its own RUs that are modulated on two different frequency bands which do not interfere together. In the rest of the paper we also consider that users covered by the micro cell are also covered by the macro cell.

\section{B. Preliminary result}

The main objective of this subsection is to establish an important preliminary result necessary to fix the implementation of the pre-scheduler. In a typical $5 \mathrm{G}$ multicell scenario in which a user may be in the coverage area of several base stations, the main problematic is to find the optimal way to share the traffic between all access points. This lies in giving a priority order to each access point in the allocation process. This priority order allows to always select the best access point to establish a connection. Three different states can describe this multicell scenario:

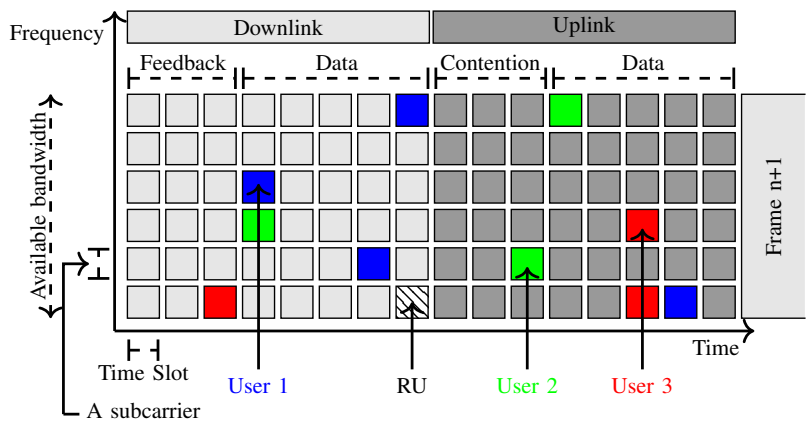

Figure 3: 5G frame structure in TDD mode

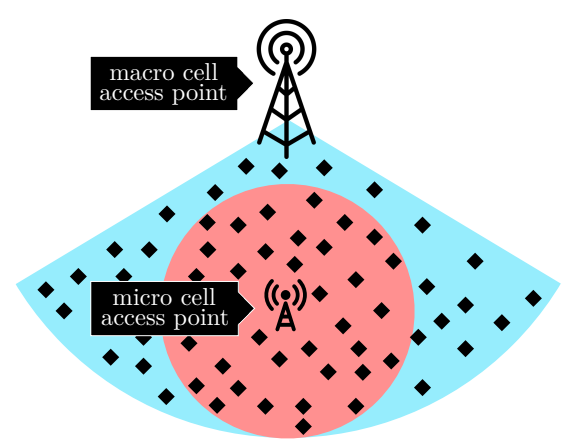

Figure 4: Random user position deployment

- $\underline{\text { State 1: }}$ : The system is not overloaded, both access points can provide RUs.

- State 2: The system is in the process of congestion. One of the two access points can't provide RUs anymore. Two different subcases are presented:

(a) The micro cell is congested, the macro cell is not.

(b) The macro cell is congested, the micro cell is not.

- State 3: The system is totally congested, no more access points can provide RUs.

As the load in a system increases, it goes from State 1 to State 2 (2(a) or (2(b)) and finally to State 3. Two allocation strategies acting during State 1 are studied. These two strategies are called "MiLP" and "MaLP" for respectively "Micro Load Priority" and "Macro Load Priority". MiLP solution prioritizes State 2(a) by allocating users to the micro in priority (if they are in its coverage area) while MaLP solution favours State 2(b) by allocating users to the macro in priority. In other words, with MiLP strategy, traditional schedulers (RR, MaxSNR,...) firstly allocate users with the micro cell access point. When this one overloads, macro cell deals with users which couldn't be assigned to the micro (due to overloading) but also with users outside micro coverage. With MaLP solution, traditional schedulers firstly allocate users with the macro cell access and then to micro cell when the first one overloads. An important result lies in finding the best allocation strategy allowing the system to be more robust to the system congestion keeping user QoE at its highest level.

In order to evaluate the performance of the two allocation strategies we decide to simulate a simple user deployment scenario. We consider a random user deployment in which some users are inside the micro cell coverage and some users 
outside. Simulation results were averaged over 100 different user position patterns. One of them is presented on Figure 4.

We also consider a variable traffic. Each user generates a traffic following an exponential distribution which fluctuates around an expectancy value common to all users. During a simulation, system load increases by adding users 2 by 2 . Base stations characteristics (frequency, power) are given in table I.

\begin{tabular}{cc|cc}
\multicolumn{2}{c|}{ Micro cell BS } & \multicolumn{2}{c}{ Macro cell BS } \\
\hline Frequency & Power & Frequency & Power \\
$26.6 \mathrm{GHz}$ & $33 \mathrm{dBm}$ & $4 \mathrm{GHz}$ & $44 \mathrm{dBm}$ \\
\hline
\end{tabular}

Table I: Base stations characteristics

Figure 5 gives the performance results in terms of global system mean packet delay (5(a)) and QoE (5(b)) for the four SoTA schedulers. Delay indicator represents the time duration between a packet creation in the backhaul network and its reception by the user equipment. A packet is considered out of delay if the time constraint imposed by the user service (streaming video, web,...) is not respected. QoE measures the user experience and is linked to the number of packets out of delay. At the end of each communication, we determine the ratio between the number of packets out of delay and the total number of packets sent to the user.

Mean delay result shows that by applying MiLP strategy, the mean delay experienced by users is more robust to the system load increase. For example, with MaxSNR scheduler, about 35 users are needed to reach a system delay of $20 \mathrm{~ms}$ with MaLP solution and up to 56 for MiLP strategy (Figure 5(a)). In other words MiLP deployment allows to accept $60 \%$ more users while guaranteeing a QoE beyond 90\% (Figure 5(b)).

By keeping the MiLP solution, the system can improve the probabilities that all users are in the radio coverage to an access point which is not overloaded. Indeed, if all microcell users are firstly assigned to the macro cell leading to its congestion (MaLP solution), a user outside the micro cell coverage can no longer receive RU from it (State 2b). It's a sub-optimal solution knowing that several users are inside micro coverage (which is not overloaded) but assigned to the macro cell. These same users should be assigned to the micro cell releasing the macro bandwidth to users that are outside the micro coverage. This result constitutes an important preliminary result before applying the MCPS prescheduler (introduced in the next section IV). In the rest of the document, MCPS pre-scheduler is implemented by considering MiLP strategy which allows to be more robust to traffic congestion.

\section{Multi-Cell Pre-Scheduler (MCPS)}

MCPS pre-scheduler objective is to avoid the system congestion by solving an allocation optimization problem when the micro cell tends to be overloaded. This situation may occur when too many users are located in the micro cell coverage area causing a high traffic peak demand leading to its congestion. In this case some traffic (i.e. some users) need to be moved to the macro cell. MCPS pre-scheduler allows to adequately choose the users located in the micro cell coverage area which are assigned to the macro cell in the allocation process since some users are more profitable to be assigned to the macro cell. MCPS allows to effectively choose these users by allocating to each one a priority index. The higher a user index is, the higher its probability to be allocated by the micro cell is. The main objective is to keep in the micro cell the more profitable users and then exclude the more profitable users for the macro (considering throughput). MCPS pre-scheduler, whose main objective is to increase system capacity, is based on users experienced throughput calculation and is described in subsection IV-A.

\section{A. Algorithm description}

MCPS pre-scheduler is based on the calculation of the users experienced throughput with the access points they can establish a connection. To each mobile $k$, a $M C P S_{k}$ is associated whose value is updated at each allocation decision instant. $M C P S_{k}$ is calculated following equation (2).

$$
M C P S_{k}=\overline{D_{k}^{\text {micro }}}-\overline{D_{k}^{\text {macro }}},
$$

where $\overline{D_{k}^{\text {micro }}}$ and $\overline{D_{k}^{\text {macro }}}$ represent the mean user $k$ experienced throughput on a RU respectively with the micro cell and the macro cell access points. So $M C P S_{k}$ values is mainly linked to users location (pathloss) and the implemented scheduler.

All users are then classified according to their $M C P S$ value with an index between 1 and $k_{\max }$ (where $k_{\max }$ is the number of users in the micro base station coverage and then concerned by $M C P S$ ). The user with higher $M C P S$ value gets index 1 and the user with lower MCPS value gets index $k_{\max }$. A positive (respectively negative) $M C P S_{k}$ value reflects the interest of connecting user $k$ to the micro cell (respectively macro cell) access point with which its experienced throughput is higher. Unfortunately, because of micro cell limited capacity, all users with a positive $M C P S$ value could not necessarily be allocated by the micro cell. Users with higher $M C P S$ value get higher index priority. The second step is then to determine the maximal number of users the micro cell can manage. This limit is calculated as follows:

$$
\operatorname{Limit}[\%]=100 \times \frac{\overline{D^{\text {micro }}} \times N_{R B}^{\text {micro }}}{\sum_{k=1}^{k_{\max }} B O_{k}(t)},
$$

where:

- $\overline{D^{\text {micro }}}[$ bit/RU] is the mean spectral efficiency of the micro cell and is measured from the previous allocations.

- $N_{R B}^{m i c r o}[R U]$ is the number of resource blocks made available by the micro cell access point and is constant.

- $\sum_{k=1}^{k_{\max }} B O_{k}(t)$ [bit] corresponds to the Buffer Occupancy of user $k$ at time $t$. The BO if the number of bit that has to be transmitted to a given user. $k_{\max }$ is the total number of users in the coverage area of the micro cell base station.

This limit corresponds to the ratio between the number of RUs the micro cell can provide and the total traffic (bits) 


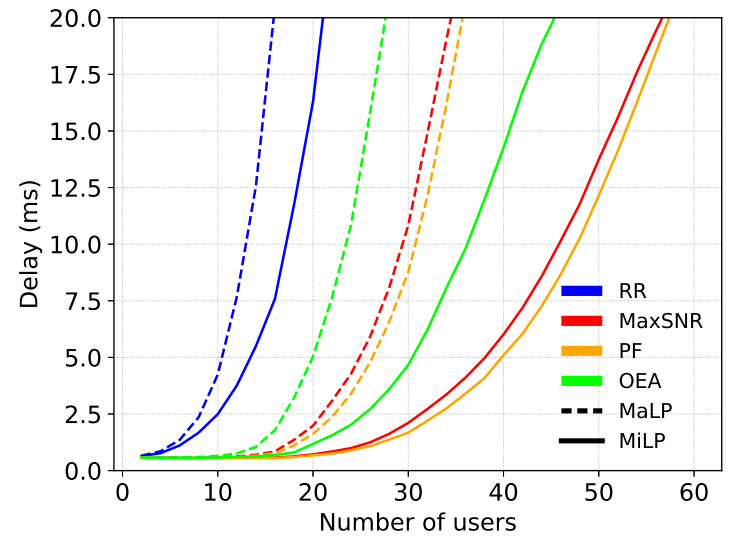

(a) Mean delay in the global system

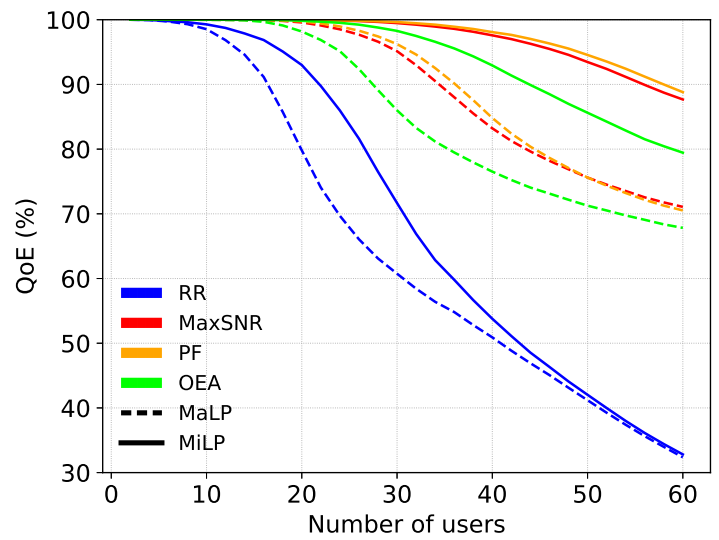

(b) Quality of Experience (QoE) indicator

Figure 5: MiLP/MaLP performance results comparison

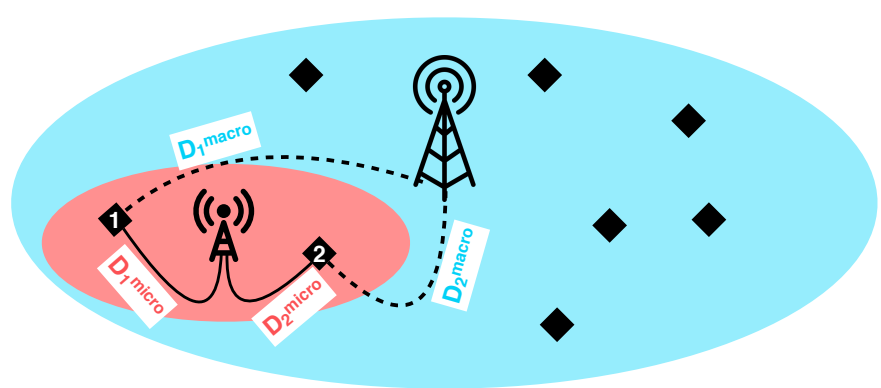

Figure 6: MCPS throughput calculation representation

created by users in the micro cell at a given time. In other words, this limit value gives the ratio of RUs the micro can handle until its congestion which corresponds to a bandwidth usage ratio equal to $100 \%$. According to this limit ratio of users, the micro cell manages the percentage of the $k_{\max }$ users that have the higher $M C P S$ value letting the macro cell the other users with lower $M C P S$. By doing so, the system allows the macro cell to allocate the users which are the most beneficial to it. So the system dynamically manages all the users by adequately arrange them between the two access points in order to increase the global system capacity. Once the MCPS has made this pre-allocation by assigning each user to an access point, traditional schedulers (RR, MaxSNR,...) take over their own assigned users in both cells. This prescheduling process is summarized by the Figure 7 with its flow chart.

\section{B. MCPS example}

In order to well understand MCPS operation, this subsection is dedicated to give an example presented on Figure 8(a). This example considers a group of 10 users all covered by both micro and macro cell access points. They are therefore concerned by MCPS pre-scheduling. In this implementation, $M C P S$ value (fourth column) is calculated from experienced throughput respectively in micro (second column) and in macro (third column). In this example, micro cell that has

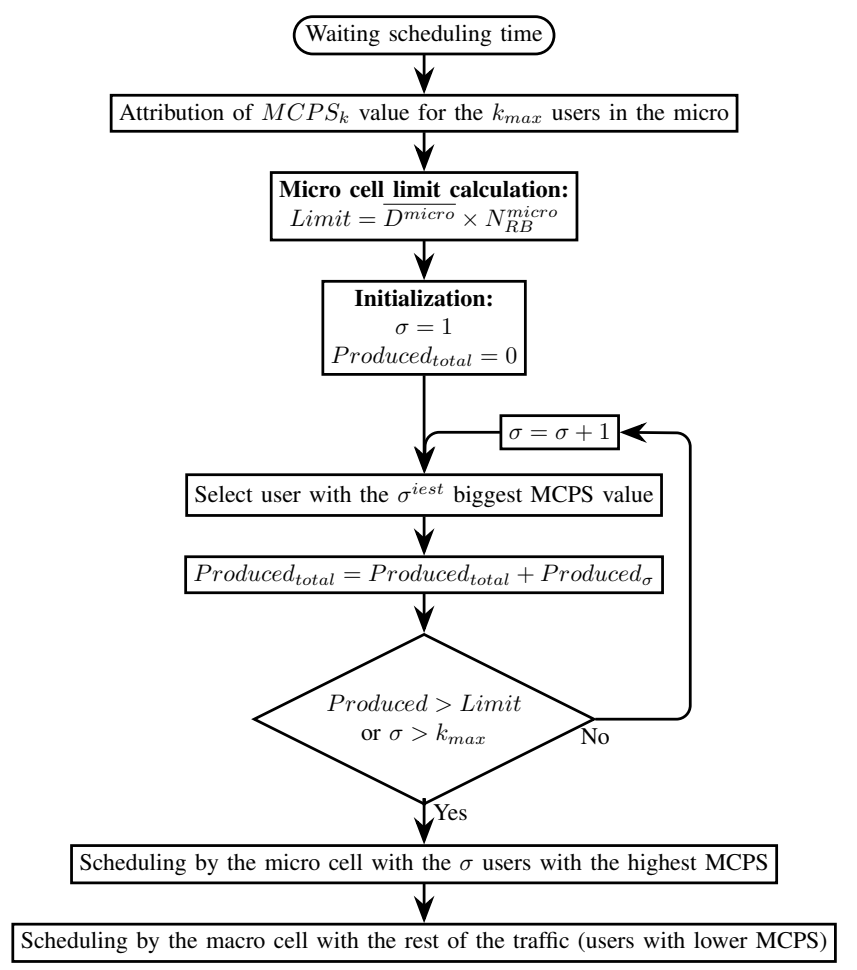

Figure 7: MCPS pre-scheduler algorithm flow chart

a fixed capacity, can provide RU to the six first users with high MCPS values (users 7, 2, 1, 5, 9 and 10). The other four users (users 3, 8, 6 and 4) with lower MCPS are allocated by the macro cell. Figure 8(b) shows the importance to sort users in order to maximize the mean system throughput. The upper table represents the previous situation 8(a) for which users are classified (MCPS applied). In this case system throughput reachs 21.8 MBit/sec. Bottom table of Figure 8(a) considers that MCPS is not applied and therefore users are not classified. So the excess traffic is randomly chosen. In this case, the mean global throughput reaches $19.5 \mathrm{MBit} / \mathrm{sec}$. It shows that MCPS users sort efficiently assigns a given user to the access point allowing him to reach a good throughput. 
Note that the MCPS limit (i.e. the number of users considered by the micro cell) is closely linked to the generated traffic at the decision-making instant. Each user produces its own variable traffic and so get a weighting that depends on what he generates. At a given time $t$, some users may generate high traffic peak while some others very stable one. This impacts the MCPS limit value and so the number of users assigned to the micro cell. Figure 8(c) gives an example of two scenarii impacted by different traffic peaks. Scenario A is a visualization of the table issued from Figure 8(a)

In scenario B, user 7 with a high MCPS value produces a high traffic peak that occupy a large portion of the microcell bandwidth. This impacts the rest of the micro cell in bandwidth availability. The result is that the number of users assigned to the micro cell may decrease. This is the case in Scenario B for which only 5 users are assigned to the micro cell. The traffic generated by the $k_{\max }$ users being totally independent to the micro cell capacity, micro cell limit is rarely fixed between two distinct users. This is the case in Scenario A with user 10 and scenario B with user 9. Their generated traffic allows to achieve $100+\epsilon \%$ of the total micro cell bandwidth. MCPS considers that, if the micro cell bandwidth usage ratio is less than $100 \%$, it assigned to it one more user by slightly exceed its maximal capacity. In that case, the micro cell capacity is fully exploited allowing to achieve better performance due to higher multi-user diversity in the micro cell. Then, the $\epsilon \%$ overflowed traffic is transferred to the macro cell.

\section{Discussion concerning MCPS value}

As explained in previous subsection IV-A, MCPS is linked to the user throughput and so closely depends on their channel condition. Experienced throughput is also linked to the implemented scheduler and on the way it favors users compared to some others. According to the channel conditions, path loss affects the user achievable throughput. If we consider a Gaussian channel without fading attenuation, $\alpha_{k, n}=1$ in equation (1) so $M C P S$ value can be written as:

$$
M C P S=\log _{2}\left(\frac{1+\eta \frac{P_{\text {micro }}}{d_{k_{\text {micro }}}^{3.5}}}{1+\eta \frac{P_{\text {macro }}}{d_{\text {macro }}^{3.5}}}\right),
$$

where $\eta$ is a constant which is common to all users and equal to $\frac{3 \times T_{s}}{2 N_{0}\left[e r f c^{-1}\left(\frac{B E R_{\text {target }, k}}{2}\right)\right]^{2}}$.

In this case, $M C P S$ mainly depends on base station radiating power and user location. Figure 9 shows that $M C P S$ value can take value from 12 (red part close to the micro cell base station) to -6 (blue part close to the macro cell base station). Circles of same $M C P S$ values are notable around micro cell base station. This phenomenon is closely linked to the distance as shown in equation 1 . Theorically locations where $M C P S=0$ represent the neutral user locations where there is no advantage to assigned the user to the micro or to the macro. We can easily show that $M C P S=0$ under the condition that $P_{\text {micro }} \times d_{k_{\text {macro }}}^{3.5}=P_{\text {macro }} \times d_{k_{\text {micro }}}^{3.5}$. This shows that assigning a user to an access point depends on its

\begin{tabular}{|c|c|c|c|c|c|}
\hline User index & $\begin{array}{c}\text { micro bit rate } \\
\mathrm{Mbit} / \mathrm{sec}\end{array}$ & $\begin{array}{c}\text { macro bit rate } \\
\mathrm{Mbit} / \mathrm{sec}\end{array}$ & MCPS value & Priority & Cell \\
\hline 7 & 30.3 & 15.2 & 15.1 & 1 & $\uparrow$ \\
\hline 2 & 33.2 & 20.9 & 12.3 & 2 & $=$ \\
\hline 1 & 20.6 & 11.8 & 8.8 & 3 & 8 \\
\hline 5 & 21.5 & 12.8 & 8.7 & 4 & . \\
\hline 9 & 31.7 & 24.6 & 7.1 & 5 & 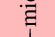 \\
\hline 10 & 18.8 & 14.9 & 3.9 & 6 & $\downarrow$ \\
\hline$\ldots$ & - & o cell limit capc & & & \\
\hline 3 & $\overline{15} . \overline{1}$ & $1 \overline{3} . \overline{0}$ & 2.10 & $\overline{7}$ & $\overline{\bar{\alpha}}$ \\
\hline 8 & 20.8 & 22.3 & -1.5 & 8 & 8 \\
\hline 6 & 8.9 & 12.3 & -3.4 & 9 & : \\
\hline 4 & 6.5 & 14.1 & -7.6 & 10 & 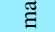 \\
\hline
\end{tabular}

(a) User classification according their MCPS value

\begin{tabular}{|c|c|c|c|c|c|c|c|c|c|c|}
\hline \multicolumn{11}{|c|}{ If users are classified : MCPS is applied } \\
\hline User index & 7 & 2 & 1 & 5 & 9 & 10 & 3 & 8 & 6 & 4 \\
\hline Realized throughput & 30.3 & 33.2 & 20.6 & 21.5 & 31.7 & 18.8 & 13.0 & 22.3 & 12.3 & 14.1 \\
\hline Total mean throughput & \multicolumn{10}{|c|}{$21.8 \mathrm{MBit} / \mathrm{sec}$} \\
\hline \multicolumn{11}{|c|}{ If users are not classified : MCPS is not applied } \\
\hline User index & 6 & 7 & 1 & 8 & 2 & 3 & 5 & 4 & 10 & 9 \\
\hline Realized throughput & 8.9 & 30.3 & 20.6 & 20.8 & 33.2 & 15.1 & 12.8 & 14.1 & 14.9 & 24.6 \\
\hline Total mean throughput & \multicolumn{10}{|c|}{ 19.5 MBit/sec } \\
\hline
\end{tabular}

(b) Impact of user classification on total system mean throughput

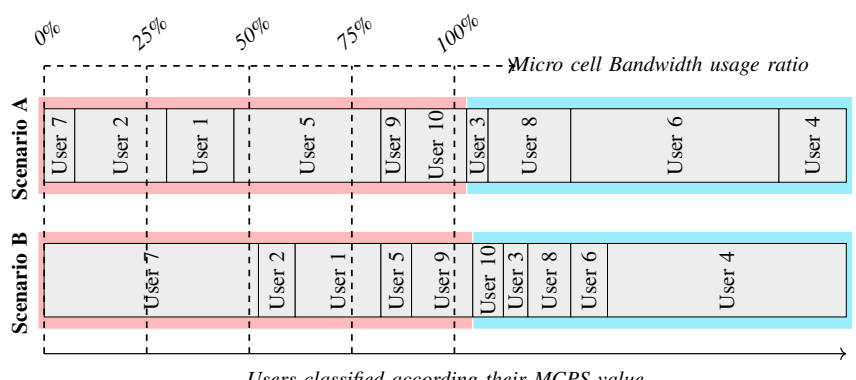

(c) Impact of VBR traffic on micro cell limit

Figure 8: MCPS example

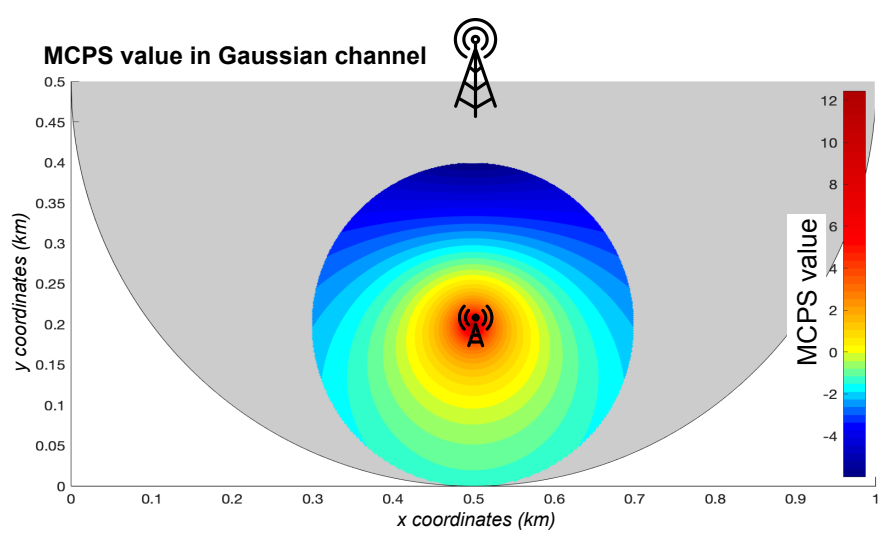

Figure 9: MCPS

location, on base stations power and also on relative position of the two access points. Some users deployments bring more benefits to MCPS pre-scheduler than some others. Indeed, given the presence of only two base stations, it is clear that MCPS value admits a symmetry behaviour along the axis of the two access points. 


\section{Performance eValuation}

\section{A. Simulation setup and studied KPIs}

This section aims to study the performance of the four SoTA schedulers, introduced in first section, when they are applied or not downstream to the $M C P S$ pre-scheduler. CoMP solutions are not considered in this part, due to architecture difference. All simulation parameters are described in section III-A and summarized in table II.

\begin{tabular}{c|c|c} 
& Micro cell BS & Macro cell BS \\
\hline Frequency & $26 \mathrm{GHz}$ & $4 \mathrm{GHz}$ \\
\hline Power & $33 \mathrm{dBm}$ & $44 \mathrm{dBm}$ \\
\hline Access Point & \multicolumn{2}{|c}{ gNodeB } \\
\hline Numerology & \multicolumn{2}{|c}{0} \\
\hline Multi-path fading & \multicolumn{2}{|c}{ Rayleigh } \\
\hline Channel model (pathloss) & \multicolumn{2}{|c}{ urban (3.5) } \\
\hline
\end{tabular}

Table II: Base stations parameters

In the two following deployment scenarios, six different Key Performance Indicators (KPI) are studied to judge the efficiency of $M C P S$ :

- The bandwidth usage ratio corresponds to the ratio between the number of RUs used and the total number of RUs made available by the access point. A ratio of 100 $\%$ underlines a congested system.

- The spectral efficiency is the quantity of transmitted information on a RU. Depending on the scheduler and its ability to use the Channel State Information (CSI) knowledge, the mean spectral efficiency may differ.

- The user capacity limit with QoE guarantee indicates the number of users the system can manage while guaranteeing a global system mean delay less than the application delay threshold.

- The system energy efficiency is a key indicator allowing to know how much a scheduler may allow a user to save energy which is essential regarding the need to improve the device battery lifetime and to reduce the carbon impact.

- The throughput represents the total number of bits distributed to the users. It grows according to the number of connected users in the system and stabilized at the maximum system capacity.

- The QoE fairness index indicates the system ability to ensure fairness regarding users requirements. This one is calculated according the following Raj Jain method [16]:

$$
J\left(\text { user }_{1}, \text { user }_{2}, \ldots, \text { user }_{n}\right)=\frac{\left(\sum_{k=0}^{n} x_{k}\right)^{2}}{n \sum_{k=0}^{n}\left(x_{k}\right)^{2}},
$$

where $x_{k}$ represents the evaluated parameter, $x_{k}=$ $Q O E_{k}$ in our case. As in the section III-B, QoE is determined with the mean ratio between the number of packets out of delay and the total number of packets.

In the next two subsections V-B and V-C, two users deployment scenarii and their respective obtained performance

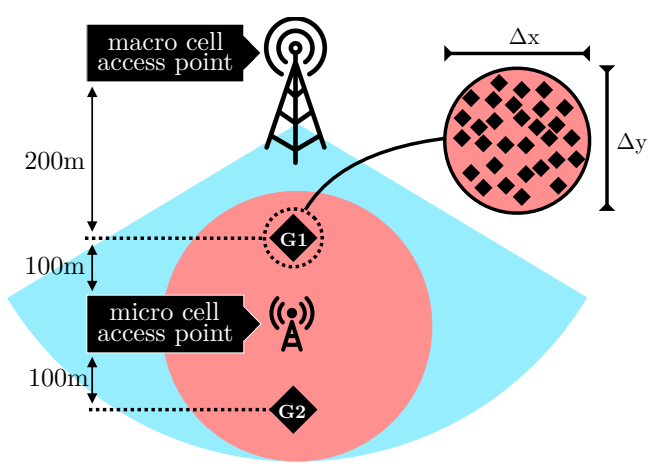

Figure 10: Grouped position deployment

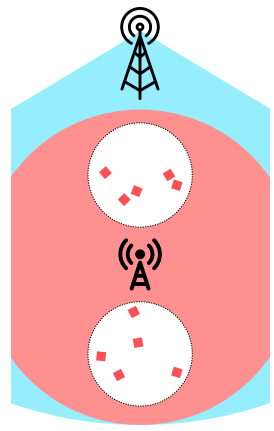

(a) 10 users

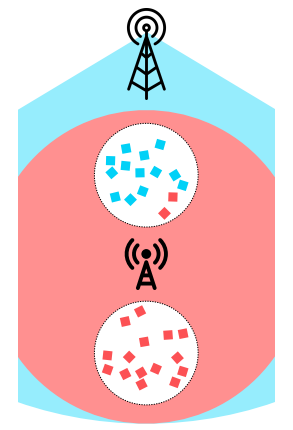

(b) 30 users

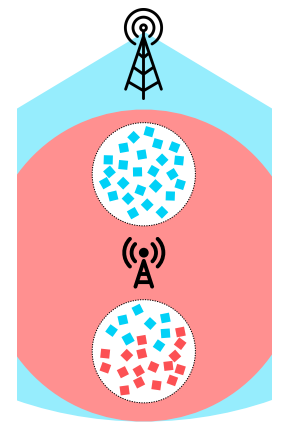

(c) 50 users
: Users allocated by macro cell

Users allocated by micro cell

Considering a micro cell maximal capacity of 17 users

Figure 11: MCPS behaviour with grouped users deployment

are presented. The base station deployment (base station power, frequency and position) remains unchanged from the preliminary results presented in subsection III-B. Performance results are obtained using discrete event simulations. The six KPIs are studied according the load in the system and more precisely following the number of users connected to the system. Application delay threshold is fixed to $100 \mathrm{~ms}$ in the following. For each discrete point, simulation results are averaged over a communication duration of 1000 Frames.

\section{B. Scenario 1: Proof of concept deployment}

In order to clearly underline the MCPS pre-scheduler behavior, we first study the simple users deployment scenario presented on Figure 10. This lies in considering two groups of users located at the same distance from the micro cell base station $(100 \mathrm{~m})$. One group is far $(400 \mathrm{~m})$ and the other one close $(200 \mathrm{~m})$ from the macro cell base station. A random position variation is assigned to each user in its group. These horizontal and vertical variations $\Delta_{x}$ and $\Delta_{y}$ follow a uniform distribution on $[-20 \mathrm{~m}, 20 \mathrm{~m}]$. This allows users to be assigned to uncorrelated fading allowing to have a better multi-user diversity. So opportunistic schedulers and MCPS can operate by always having a wide range of choices. We also consider that each user generates a realistic variable traffic $[17,18]$.

First results are exposed on Figure 12 and present the performance of the studied deployment scenario for the four 


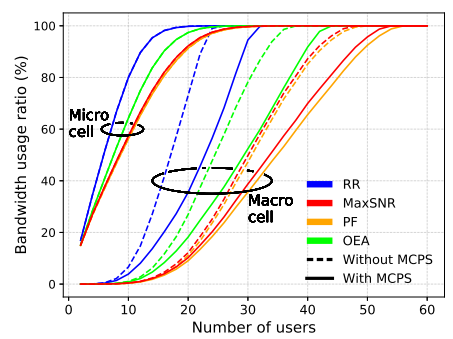

(a) Bandwidth usage ratio

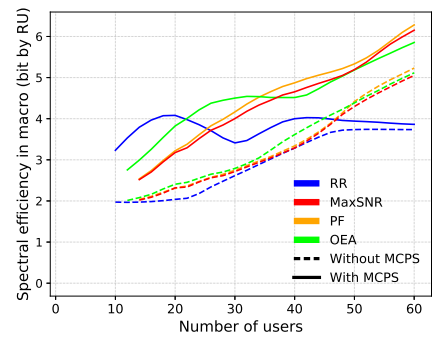

(d) Spectral efficiency in the macro cell

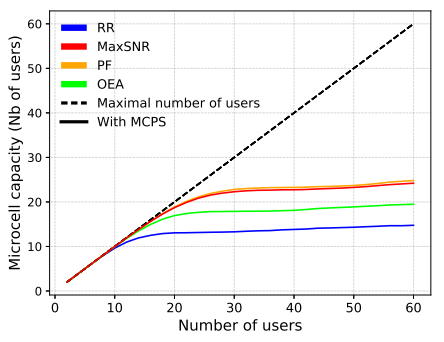

(b) Micro cell users capacity

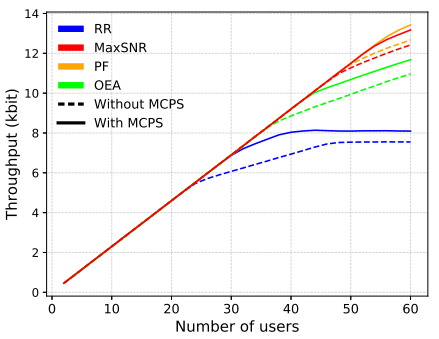

(e) Throughput

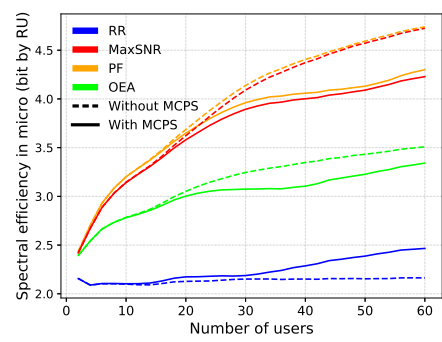

(c) Spectral efficiency in the micro cell

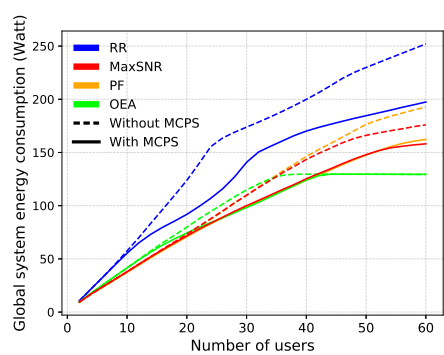

(f) System energy consumption

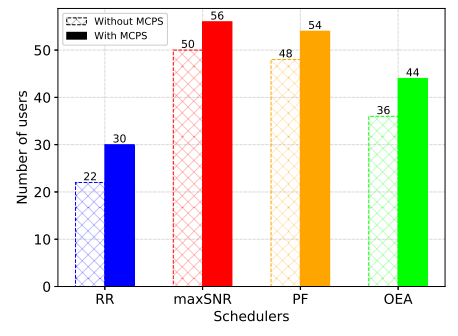

(g) User capacity limit with QoE guarantee

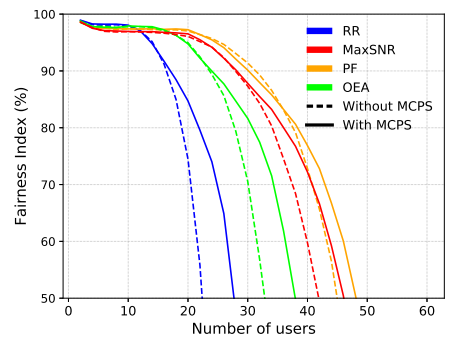

(h) Fairness Index

Figure 12: Performance results for scenario 1

SoTA schedulers when they are applied (full line), or not (dashed line) downstream to our MCPS pre-scheduling solution. In order to well understand the system behaviour facing the increase of the traffic load, bandwidth usage ratio graph (Figure 12(a)) gives the result both for micro and macro cells. We consider that the full system (micro+macro) is totally congested when both cells admit a bandwidth usage ratio of $100 \%$. Before starting any technical explanation, it is important to remember that MiLP allocation strategy has been implemented: users are firstly assigned to micro cell, if possible, before the macro cell.

Concerning the bandwidth usage ratio, results (Figure 12(a)) highlights that the global system resists to a higher load (higher number of users) when the MCPS pre-scheduler is applied. With MCPS, schedulers are able to absorb more traffic and are more robust to unexpected traffic peaks. For example MCPS allows Round Robin to accept 24 users in the system without MCPS and 32 with MCPS. This lead to accept $33.3 \%$ more users with MCPS. In the same way MCPS allows the system to deal with $17.4 \%$ more users with MaxSNR. Figure 12(b) is linked to this result and shows the number of users considered by schedulers in the micro cell when applied with MCPS (and in dashed line without MCPS). For very low traffic loads the total numbers of users considered by the micro cell is equal to the total number of users in the system. When traffic load increase, the MCPS attributes the most beneficial users respectively to the micro and macro cell. Maintaining the maximum number of user manageable by the micro, curve flat appears when micro cell reach maximum capacity and macro begins to manage the overloaded traffic. The asymptotic behavior testifies of the maximal number of users the micro cell can manage depending of the scheduler.

Bandwidth usage behaviour is closely linked to the spectral efficiency one. By applying MCPS pre-scheduler, a significant decrease of the spectral efficiency for opportunistic schedulers (MaxSNR, PF, OEA) is notable in the micro cell (Figure 12(c)). This loss in the micro cell is mainly due to this type of scheduler that need a high multi-users diversity in order to always choose the best user allowing to offer the highest system capacity. By unloading some users to the macro cell (case (b) on Figure 11), MCPS pre-scheduler restrains the optimal operation of schedulers when selecting the best user. This degradation is perceptible from a given number of users $(\simeq 14$ for $\mathrm{OEA}, \simeq 16 \mathrm{MaxSNR}$ and $\mathrm{PF})$, that underlines 


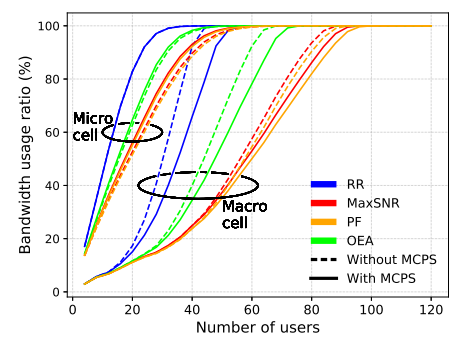

(a) Bandwidth usage ratio

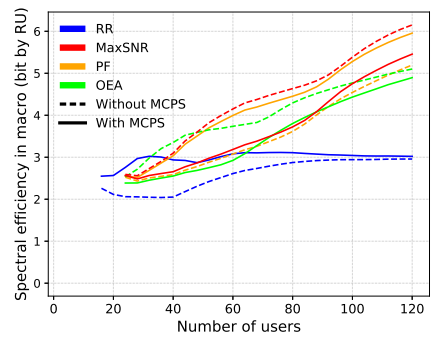

(d) Spectral efficiency in the macro cell

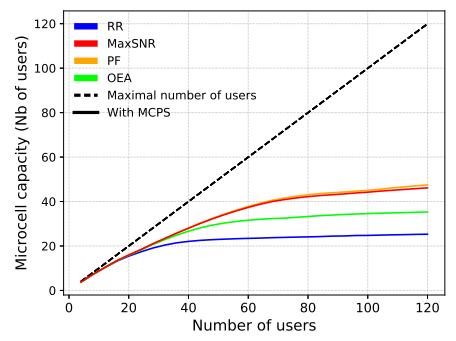

(b) Micro cell users support

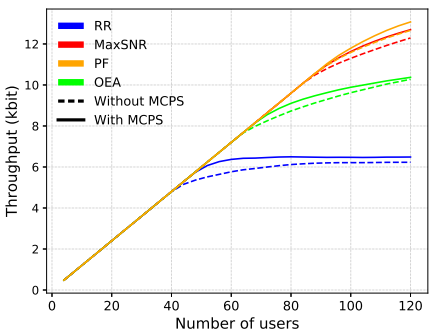

(e) Throughput

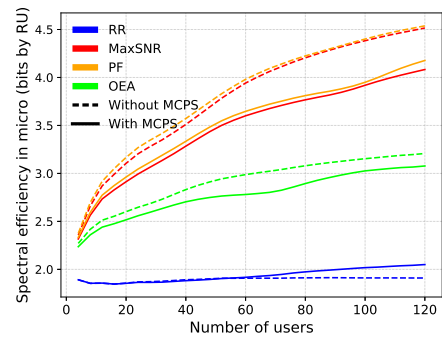

(c) Spectral efficiency in the micro cell

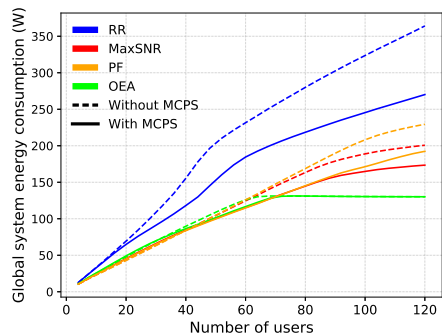

(f) System energy consumption

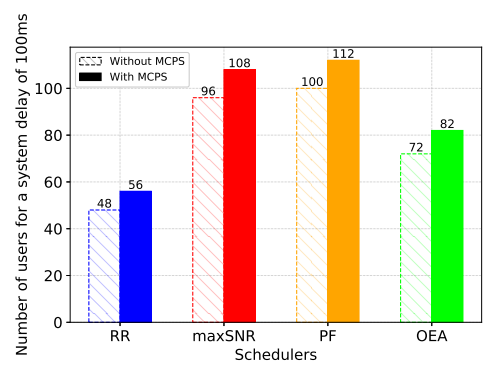

(g) User capacity limit with QoE guarantee

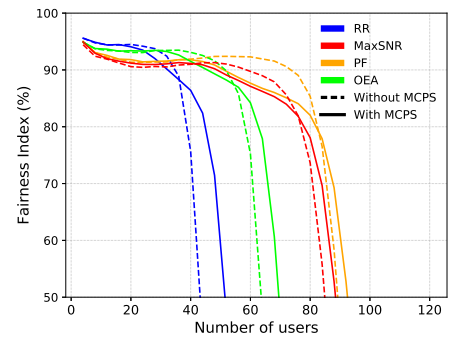

(h) Index Fairness

Figure 13: Performance results for scenario 2

when MCPS begins to operates. Indeed, below this number of users, congestion probabilities are negligible and MCPS is inactive. Round Robin which is a non opportunistic scheduler admits a slight gain in the micro by applying MCPS. Round Robin does not take into account channel conditions and user diversity. By applying MCPS with Round Robin, the loss of multi-user diversity has therefore no consequence on spectral efficiency. The impact of MCPS is even positive on spectral efficiency. Indeed, according to Figure 11(b) when the micro cell saturates, close users from the macro access point (and the farthest to the micro) are the first assigned to the macro (MCPS behaviour). Users staying in the micro cell admit a higher mean achievable throughput due to better path loss, spectral efficiency for RR increases in the micro cell.

However, this micro cell spectral efficiency loss is largely compensated by a gain in the macro cell (Figure 12(d)). As soon as the load exceeds a given threshold specific to each scheduler, MCPS detects the micro cell congestion risk and adequately share the most beneficial users between micro and macro cell macro cell. This threshold is reached when the micro cell has managed the number of users corresponding to its maximal capacity (Figure 12(b)). Macro cell spectral efficiency behaviour can be divided into four parts:
- For very low loads ( $<10$ users), system is widely underloaded and macro spectral efficiency does not exist since all users are allocated by the micro cell (Figure11(a)).

- When the micro cell overloads, first users that are assigned to the macro cell are the one with good throughput (close users - low MCPS). Spectral efficiency in the macro increases (Figure11(b)). The spectral efficiency gain with MCPS is linked to a better management of users. Macro cell receives the more profitable users to it.

- When the micro reached its maximal users capacity, the macro cell has to manage far users, which are less advantageous in terms of throughput. This slowdown is noticeable on Figure 12(d) from 20 to 32 users for RR, 30 to 42 users for OEA, and from 40 to 50 users for MaxSNR and PF. The curve with MCPS solution tends to get closer from the curve without MCPS solution (compared to Figure11(d)). But the increase still persists due to a better users-cells repartition.

- For very high loads this gap seems to be maintained or even increased. With MCPS, opportunistic schedulers resume their normal operation reaching good performance due to the supplementary of multi-users diversity (Figure11(e)). A significant throughput gain with MCPS 


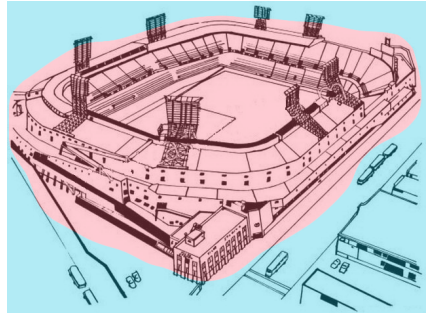

(a) Realistic stadium deployment

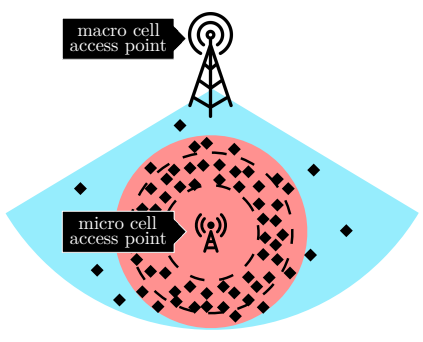

(b) dessin
Figure 14: Realistic scenario representation

is obtain over non-MCPS solutions thanks to more close users assignation.

Figure 12(e) aggregates previous results and shows the global reachable throughput by each solution in the whole system. It underlines that all schedulers increase their system capacity limit with MCPS.

In addition to increase the overall capacity of the system (bandwidth usage ratio and spectral efficiency previously studied), some additional KPI are improved by applying MCPS pre-scheduler (fairness, energy saving). For example Figure 12(f) shows that the global system energy consumption are improved with MCPS. Indeed, MCPS effectively assigned users with the access point they can achieve a good throughput and therefore they are able to finish their connection quicker. Users are then in an active mode for a shorter time and then consume less.

Figure 12(h) also shows the MCPS ability to keep QoE fairness between users as the load increases. Considering MaxSNR scheduler at 40 users in the system, the fairness index reaches $60 \%$ and $72 \%$ respectively without and with MCPS. This gain is perceptible for the four schedulers. When users are assigned to base station, they share the access point with other users whose throughput difference is not significant. More users obtain a good QoE satisfying their application requirements $(12(\mathrm{~g}))$.

These first simulation results constitute a proof of concept testifying the performance bring by MCPS pre-scheduling. A more realistic deployment is studied in the next subsection V-C.

\section{Scenario 2: Realistic user deployment}

In order to confirm the previous performance results bring by MCPS pre-scheduling, we simulate a more realistic users deployment scenario. This deployment is inspired by $3 \mathrm{GPP}$ deployment specifications [19] and is represented on Figure 14. We consider an ultra-dense deployment scenario for which a user group is distributed around an access point. This access point allows to unload the macro cell in this dense area where the demand is high. Typically, we can consider a stadium deployment (Football stadium, concert arena). A stadium is typically a place where the traffic is high (video uploading,...) and where a micro cell hotspot deployment makes sense. This deployment represents a concrete case for which MCPS pre-scheduler can bring a benefit compared to a traditional solution. This realistic deployment considers a hotspot located in the center of the stadium (Figure 14(a)) providing high data rate broadband coverage to the users located around it. We evaluate the MCPS performance by studying the same KPIs than previous users deployment. In order to get reliable KPIs, simulation results were average over 100 different users deployment patterns. The traffic generated by users and base stations characteristics, remains the same as previous studies. Results are presented on Figure 13.

According to Figure 13 this scenario confirms MCPS prescheduler performance. Results concerning bandwidth usage ratio show that the system can allocate $9.6 \%$ more users for MaxSNR and $16 \%$ more for RR when applying MCPS. We can also note the same spectral efficiency trends, meaning a loss in the micro cell compensated by a gain in the macro. User QoE (Figure 13(g)) guarantees and QoE fairness (13(h)) are also improved.

Figure 13 (b) shows that the numbers of users considered by the micro cell is always lower than the total number of users in the system. This shows that some users are located outside the micro cell base station coverage who necessarily have to be allocated by the macro cell access point. We also note the same asymptotic behavior testifying the micro cell maximal capacity and so the number of users the micro cell can manage. The two scenarios previously studied show the benefit brings by MCPS pre-scheduler in a multicell scenario.

\section{CONCLUSION}

In the literature many research efforts have been done focusing on scheduling optimization in a simple deployment scenario with one cell. However these scheduling techniques are often not optimized to a multicell scenario. This paper proposed a new pre-scheduling solution called "MCPS" for multicell wireless networks. Taking into account users achievable throughput, MCPS effectively share the traffic load between the different access points increasing the global system capacity. Performance results have shown the benefits bring by this new approach allowing the system to be more robust to the congestion and traffic peaks. Future work will focus on improving the MCPS solution towards a service-oriented pre-scheduler taking into account users service parameters allowing to better adapt the allocation process to users needs (generated traffics, service time constraints,...).

\section{REFERENCES}

[1] Call:H2020-ICT-2016-2, "Deliverable d2.1 scenarios, kpis, use cases and baseline system evaluation," E2E-aware Optimizations and advancements for Network Edge of $5 G$ New Radio (ONE5G), Dec 2016.

[2] O. Bulakci, A. Kaloxylos, J. Eichinger, and C. Zhou, "Ran moderation in $5 \mathrm{~g}$ dynamic radio topology," in 2017 IEEE 85th Vehicular Technology Conference (VTC Spring), 2017, pp. 1-4.

[3] Y.-H. Chen, H.-L. Chao, S.-H. Wu, and C.-H. Gan, "Resource allocation with CoMP transmission in ultra dense cloud-based LTE small cell networks," in 2017 IEEE 28th Annual International Symposium on Personal, Indoor, and Mobile Radio Communications (PIMRC). Montreal, QC: IEEE, Oct. 2017, pp. 1-5.

[4] L. Liu, Y. Zhou, V. Garcia, L. Tian, and J. Shi, "Load Aware Joint CoMP Clustering and Inter-Cell Resource Scheduling in Heterogeneous Ultra Dense Cellular Networks," IEEE Transactions on Vehicular Technology, vol. 67, no. 3, pp. 2741-2755, Mar. 2018. 
[5] M. Minelli, M. Ma, M. Coupechoux, and P. Godlewski, "Scheduling impact on the performance of relay-enhanced lte-a networks," IEEE Transactions on Vehicular Technology, vol. 65, no. 4, pp. 2496-2508, April 2016.

[6] Cheong Yui Wong, R. S. Cheng, K. B. Lataief, and R. D. Murch, "Multiuser ofdm with adaptive subcarrier, bit, and power allocation," IEEE Journal on Selected Areas in Communications, vol. 17, no. 10, pp. 1747-1758, Oct 1999.

[7] H. Kim, K. Kim, Y. Han, and S. Yun, "A proportional fair scheduling for multicarrier transmission systems," Proc. IEEE Int. Vehicular Technology Conference (VTC), vol. 2, pp. 409 - 413, Sept 2004.

[8] X. Ge, H. Jin, and V. C. M. Leung, "Cdf-based scheduling algorithm for proportional throughput fairness," IEEE Communications Letters, vol. 20, no. 5, pp. 1034-1037, May 2016.

[9] C. Gueguen, "Opportunistic Energy Aware Scheduler for Wireless Networks," 2013 IEEE 77th Vehicular Technology Conference (VTC Spring), pp. 1-5, Jun. 2013.

[10] C. Gueguen and M. Manini, "Dynamic tradeoff between energy and throughput in wireless 5g networks," Wireless Communications and Mobile Computing, vol. 2018, 2018.

[11] S.-Y. Kim and C.-H. Cho, "Call Blocking Probability and Effective Throughput for Call Admission Control of CoMP Joint Transmission," IEEE Transactions on Vehicular Technology, pp. 1-1, 2016.

[12] T. M. Shami, D. Grace, A. Burr, and M. D. Zakaria, "User-centric JTCoMP clustering in a 5G cell-less architecture," in 2018 IEEE 29th Annual International Symposium on Personal, Indoor and Mobile Radio Communications (PIMRC). Bologna, Italy: IEEE, Sep. 2018, pp. 177181.

[13] D. Lopez-Perez, M. Ding, H. Claussen, and A. H. Jafari, "Towards 1 Gbps/UE in Cellular Systems: Understanding Ultra-Dense Small Cell Deployments," IEEE Communications Surveys \& Tutorials, vol. 17, no. 4, pp. 2078-2101, 2015.

[14] M. Andrews, K. Kumaran, K. Ramanan, A. Stolyar, P. Whiting, and R. Vijayakumar, "Providing quality of service over a shared wireless link," IEEE Communications Magazine, vol. 39, no. 2, pp. 150-154, Feb 2001.

[15] J. D. Parsons, The Mobile Radio Propagation Channel. Wiley, 1992.

[16] H. SHI, R. V. Prasad, E. Onur, and I. G. M. M. Niemegeers, "Fairness in wireless networks:issues, measures and challenges," IEEE Communications Surveys Tutorials, vol. 16, no. 1, pp. 5-24, First 2014.

[17] S. Baey, "Modeling mpeg4 video traffic based on a customization of the dbmap," in Proc. Int. Symposium on Performance Evaluation of Computer and Telecommunication Systems (SPECTS), 2004, pp. 705714.

[18] A. K. A. Tamimi, R. Jain, and C. So-In, "Modeling and prediction of high defninition video traffic: A real-world case study," in Proc. Second International Conferences on Advances in Multimedia, June 2010, pp. $168-173$.

[19] E. 3rd Generation Partnership Project, 5G; Service requirements for next generation new services and markets, 3GPP TS 22.261 version 15.5.0 Release 15, 2018-07. 